\title{
Medium Access Cooperations for Improving VoIP Capacity over Hybrid 802.16/802.11 Cognitive Radio Networks
}

\author{
Deyun $\mathrm{Gao}^{1}$, Jianfei $\mathrm{Cai}^{2}$, and Chuan Heng $\mathrm{Foh}^{2}$ \\ ${ }^{1}$ School of Electronics and Information Engineering, Beijing Jiaotong University, Beijing \\ 100044, P.R. China \\ ${ }^{2}$ School of Computer Engineering, Nanyang Technological University, \\ Singapore 639798
}

\begin{abstract}
There are some existing works that study the coexistence of 802.16 and 802.11 networks. However, not many of them consider the resource allocation issues in the case of delivering traffic between mobile stations and Internet users through an access point (AP) and a base station (BS) which operate at the same frequency band. In this paper, we design a cooperation mechanism for 802.16 and 802.11 to share the same medium with adaptable resource allocation. The adaptiveness in resource allocation in our design eliminates the potential inefficiency from the cooperation due to bandwidth bottleneck. Targeting VoIP applications, we propose a simple approach for the adaptation that optimizes the resource allocation not only between the IEEE 802.11 AP and the stations but also between the IEEE 802.16 BS and the IEEE 802.11 AP. Numerical results show significant improvement in voice capacity.
\end{abstract}

\section{Introduction}

There have been tremendous advances in wireless networks and mobile devices in recent years. It is expected that different radio technologies, including WiFi (IEEE 802.11), UWB (IEEE 802.15.3), WiMAX (IEEE 802.16), ZigBee (IEEE 802.15.4), Bluetooth and $3 \mathrm{G}$ will coexist. With such a rapid growth of wireless technologies, spectrum scarcity has become a serious problem as more and more wireless applications compete for very little spectrum. In order to solve this problem, the cognitive radio technology was introduced in the late 1990s by Joseph Mitola [1]. Although the cognitive radio technology sheds light on spectral reuse, it leaves open the issues of how to efficiently and practically deploy cognitive radios [2]. Recently, cognitive radio has attracted a lot of interests from research community [3 4], where dynamic spectrum utilization is the main focus.

Among various wireless networks, 802.16 and 802.11 are the two most important wireless access technologies, which could be integrated to provide Internet connections for end users as described in [5]6]. In general, 802.16 can provide wireless backhaul connectivity to homes and offices while 802.11 offers complementary local area connectivity such as within a home, an office or a campus. In this paper, we consider hybrid 802.11 and 802.16 cognitive radio networks. Fig. 1 shows a typical scenario, where

A. Das et al. (Eds.): NETWORKING 2008, LNCS 4982, pp. 889 900, 2008.

(C) IFIP International Federation for Information Processing 2008 


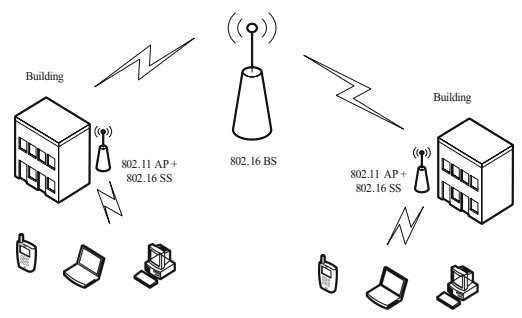

Fig. 1. A typical scenario of hybrid 802.16 and 802.11 networks

mobile stations are connected with 802.11 APs and the APs are connected to Internet through an 802.16 BS. Under such a scenario, 802.16 and 802.11 might have to share the spectrum, e.g., the U-NII frequency band at $5 \mathrm{GHz}$ that could be used by both 802.11a and 802.16a.

Currently, in the unlicensed bands, only a few spectrum sharing methods [7 89] have been proposed. In [7], Berlemann et al. proposed to partially block 802.11 stations to access the medium so that 802.16 could use the same spectrum. In [8], Jing et al. proposed to utilize the available degrees of freedom in frequency, power and time, and react to the observations in these dimensions to avoid interference. In [9], Jing and Raychaudhuri proposed to use a common spectrum coordination channel to exchange the control information in order to cooperatively adapt the key PHY-layer parameters such as frequency and power. All of these existing schemes do not consider the resource allocation issues in the case of delivering traffic between mobile stations and Internet users through an AP and a BS, which are sharing the same frequency band. In this paper, we design a cooperation mechanism for 802.16 and 802.11 to share the same medium with adaptable resource allocation. We further consider VoIP applications and propose a simple approach to find the optimal resource allocation not only between the AP and the stations but also between 802.16 and 802.11 .

The rest of the paper is organized as follows. In Section 2 we give a brief overview of the 802.11 and 802.16 MAC protocols. In Section 3, we propose the medium access cooperation mechanism to coordinate the channel access between 802.11 and 802.16. In Section 4, we discuss how to optimally allocate the resource for VoIP over the hybrid 802.16/802.11 networks. Numerical results are provided in Section 5 and conclusions are given in Section 6

\section{Overview of 802.11 and 802.16 MAC Protocols}

\subsection{11 MAC Protocol}

In 802.11 WLANs, the MAC layer defines the procedures for 802.11 stations to share a common radio channel. The legacy 802.11 standard specifies the mandatory distributed coordination function (DCF) and the optional point coordination function (PCF) [10]. DCF is essentially a "listen-before-talk" scheme based on CSMA/CA, while PCF uses polling to provide contention-free transmission. To enhance the QoS supports in 802.11, 
the new IEEE 802.11e [11] introduces the hybrid coordination function (HCF), which includes two medium access mechanisms: enhanced distributed channel access (EDCA), and HCF controlled channel access (HCCA), which can be regarded as the extensions of DCF and PCF, respectively.

In the IEEE 802.11 MAC protocol, time is divided into superframes, where each superframe consists of two types of phases: contention free period (CFP) and contention period (CP). In the legacy 802.11, DCF is used in CPs and PCF is used in CFPs. Likewise, in the 802.11e MAC protocol, EDCA can only be used in CPs, while HCCA can be used in both phases. Fig. 2 illustrates the different periods under HCF. Note that the CAP (controlled access phase) is defined as the time period that the medium control is centralized. It can be seen that CAPs consist of not only CFPs but also parts of CPs.

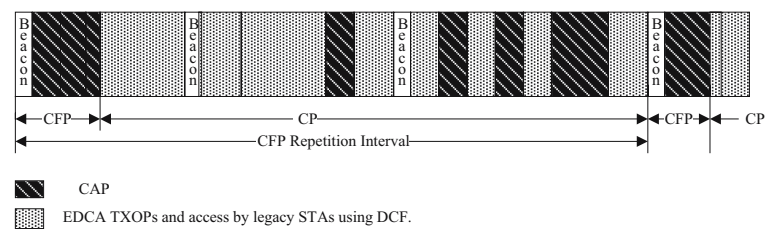

Fig. 2. An example of CAPs/CFPs/CPs

In this research, we consider EDCA is used in WLANs for the communications between mobile stations and the access point (AP). The EDCA mechanism extends the legacy DCF through introducing multiple access categories (ACs) to serve different types of traffics. In particular, there are four ACs with independent transmission queues in each mobile station. The four ACs starting from AC3 to AC0 are designed to serve voice traffic, video traffic, best effort traffic, and background traffic, respectively. Each AC, basically an enhanced variant of DCF, contends for transmission opportunities (TXOPs) using one set of the EDCA channel access parameters. The major parameters include

- $C W \min [A C]$ : minimal contention window $(\mathrm{CW})$ value for a given $\mathrm{AC}$.

- $C W \max [A C]$ : maximal $\mathrm{CW}$ value for a given $\mathrm{AC}$.

- AIF $S[A C]$ : arbitration interframe space. Each AC starts its backoff procedure after the channel is idle for a period of $A I F S[A C]$.

- TXOPlimit $[A C]$ : the limit of consecutive transmission. During a TXOP, a station is allowed to transmit multiple data frames but limited by $T X O P l i m i t[A C]$.

\subsection{16 MAC Protocol}

The 802.16 MAC protocol [12] supports point to multipoint (PMP) and mesh network modes, schedules the usage of the air link resource and provides QoS differentiations. In this paper, we focus on the PMP mode, where one base station (BS) and many subscriber stations (SSs) form a cell similar to that in cellular networks. There are two types of duplexing schemes: FDD (Frequency Division Duplex) and TDD (Time Division Duplex). Most WiMAX implementations mainly use TDD. 
Fig. 3 shows the frame structure in a typical 802.16 TDD system. Basically, time is divided into frames, and each frame consists of uplink and downlink subframes. A downlink subframe (DL-Subframe) has two major parts: control information and data. There are two important maps in the control information of a DL-Subframe: DL-MAP and UL-MAP, which describe the slot locations for the downlink and uplink subframes. It is through the DL-MAP and UL-MAP fields that the BS allocates resources to SSs. The UL subframe contains an initial ranging field, a bandwidth request field, and burst fields for MAC PDUs. The 802.16 MAC protocol supports both polling and contentionbased mechanisms for SSs to send bandwidth requests.

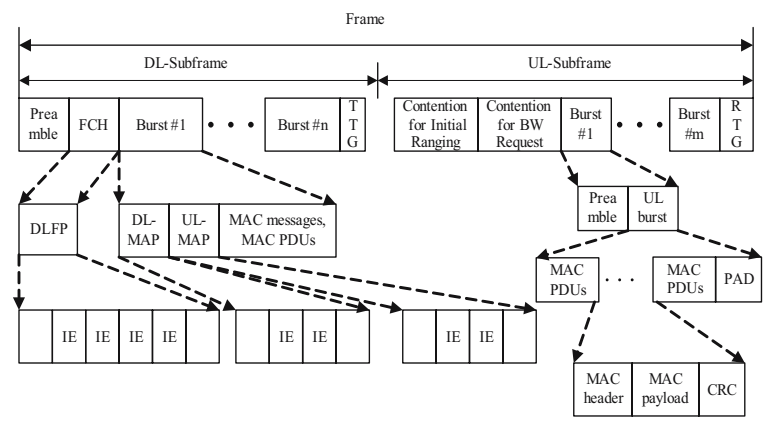

Fig. 3. The frame structure of 802.16

The 802.16 MAC protocol is connection-oriented. The QoS requirements of a connection in a SS can be varied by sending requests to the BS. Service differentiation has also been introduced in WiMAX [13], where four service classes are defined:

- Unsolicited grant service (UGS) for CBR traffic such as voice

- Real-Time polling service (rtPS) for real-time VBR traffic such as MPEG videos

- Non-realtime polling service (nrtPS) for non-realtime traffic such as FTP

- Best effort (BE).

\section{Medium Access Cooperations in hybrid 802.16/802.11 networks}

In this paper, we consider a typical scenario of hybrid 802.16/802.11, where an 802.16 BS is connecting to a few SSs using TDMA/TDD and each SS is an AP communicating with many mobile stations through EDCA. Although the medium access protocols in both 802.16 and 802.11 have been well defined, we still need to design a mechanism to coordinate the medium access between them in order for them to operate at the same spectrum.

Since a typical superframe in 802.11 is about 100 - $200 \mathrm{~ms}$, much longer than a frame in 802.16 (typically $5-20 \mathrm{~ms}$ ), hence it is a natural choice to embed 802.16 frames into an 802.11e superframe and use CAPs for the communications between APs/SSs and the BS. In particular, when an AP/SS joins into 802.16, the BS periodically allocates some time slots in each frame to the AP/SS. The AP/SS can obtain the frame length 
information from the frame header. After that, the AP/SS uses the HCCA mechanism to send one packet such as RTS to inform all the mobile stations the periodic time intervals of the 802.16 frames indicated by network allocation vector (NAV), as shown in Fig. 4. All the mobile stations and the AP will not communicate each other during the periods indicated by NAVs, while for other periods they communicate using EDCA. In this way, we avoid transmission conflictions between 802.16 and 802.11.

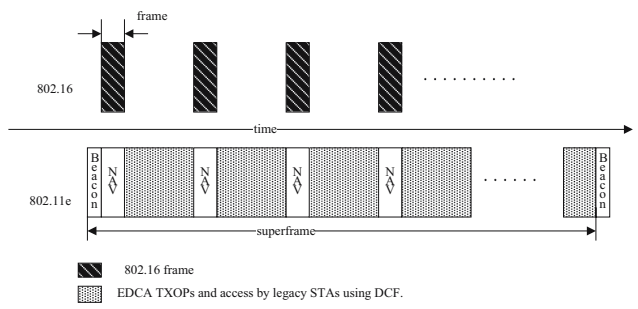

Fig. 4. Medium access cooperations between 802.16 and 802.11

In 802.16, when the traffic conditions change, we need to change the 802.16 frame length to accommodate the new traffic load. All the attached APs/SSs need to send a new NAV to their associated stations. We would also like to point out that the differentiated services in both 802.11 e and 802.16 are quite similar. We could directly map each of the four services in 802.11e into one of the services in 802.16 although the ways of implementing the service differentiation are different, where the former is through adjusting the EDCA parameters and the latter uses prioritized scheduling.

Note that, our proposed scheme also applies to multiple WLAN cells each of that connects to one SS. We assume that these WLAN cells do not located within the interference range. The interference problems between WLAN cells are outside of the focus of this paper. Under such scenario, 802.16 BS needs to chose the maximum transmission time requirements among these WLAN cells as the common requirement. Then, 802.16 BS allocates some time slots that satisfy the common requirement to each AP/SS. Each WLAN cell can complete the data transmission in parallel during the allocated time slots.

\section{Improved Resource Allocation for VoIP Applications}

Considering VoIP applications in the hybrid 802.16/802.11 networks, each voice talk involves one 802.11 mobile user and another user connected to Internet, and the communications go through one AP and one BS. One of the most important issues is how to optimally allocate the resource among mobile stations, AP and BS so that more VoIP connections can be supported.

In our previous work [14], we have studied the case of VoIP over WLANs. We found that the AP is the bottleneck for VoIP applications if we directly use the legacy DCF mechanism. We proposed to use EDCA to solve the bottleneck problem. In particular, we give the AP higher priority than mobile stations. The experimental results in [14] shows the improved voice capacity. 
For VoIP over the hybrid 802.16/802.11 networks, the bottleneck problem of AP becomes even severe since it needs to transmit not only all the 802.16 downlink packets to the stations but also all the 802.11 uplink packets to the BS.

\subsection{Improved Resource Allocation}

In order to appropriately allocate resource, we need to consider the four throughput: the uplink and the downlink throughput of 802.16 denoted as $S_{u p}^{16}$ and $S_{d w}^{16}$. We further define $S_{u p}^{11}$ and $S_{d w}^{11}$ to be the uplink throughput of EDCA from each station and the downlink throughput of EDCA from the AP respectively. For simplicity, we assume there is only one SS. The following derivation can be easily extended to the case of multiple SSs.

Considering the symmetric property of VoIP traffic, the contention-free resource allocation in 802.16 and contention-based resource allocation in EDCA, we have

$$
\begin{array}{r}
S_{u p}^{16}=S_{d w}^{16} \\
S_{u p}^{16}=N R_{r e q} \\
S_{u p}^{11}(1-r) \geq R_{r e q} \\
S_{d w}^{11}(1-r) \geq N R_{r e q},
\end{array}
$$

where $N$ is the number of voice connections, $R_{r e q}$ is the one-way voice throughput requirement, and $r$ is the time fraction occupied by 802.16. The adjustable parameters include all of the EDCA parameters such as CWmax, CWmin, retry limits, and others. In this research, we only consider adjusting the CWmin of the AP/SS and fix all the other EDCA parameters.

The condition for optimal operation can be described as follows.

$$
\left\{\begin{aligned}
\text { Maximize } & N \in \mathbb{N} \\
\text { subject to } & (r-1)\left(N S_{u p}^{16}\left(N, W_{d w}\right)+S_{d w}^{16}\left(N, W_{d w}\right)\right) \\
& +r\left(S_{u p}^{16}(N)+S_{d w}^{16}(N)\right) \leq B
\end{aligned}\right.
$$

where $B$ is the total bandwidth for sharing between 802.16 and 802.11 . Since all throughput functions, namely $S_{u p}^{16}(N), S_{d w}^{16}(N), S_{u p}^{11}\left(N, W_{d w}\right)$ and $S_{d w}^{11}\left(N, W_{d w}\right)$, are monotonically increasing functions in terms of $N$ where $N \in \mathbb{N}$, the solution can be practically computed numerically by searching for $N_{\max }$ with the following method.

Step 1: Set $N$ to a small initial value.

Step 2: Calculate the aggregate one-way voice traffic load. Then, according to the first two equations in (1), we obtain $S_{u p}^{16}$ and $S_{d w}^{16}$. Based on 802.16 frame structure, we can compute the length of an 802.16 frame (see Section 4.3). Further considering the proposed setup between 802.16 frames and an EDCA superframe shown in Fig. 4 we derive $r$.

Step 3: Based on the obtained $r$ value, we test different values of $W_{d w}$, where $W_{d w}=C W_{\min }[d w]+1$. If we can find a particular $W_{d w}$, for which the corresponding uplink and downlink saturation throughput (see Section 4.2) can satisfy the throughput requirements shown in the two inequalities in (1), we set $N=N+1$ and go back 
to step 2. Otherwise, we stop and set $N_{\max }=N-1$. Note that we use the EDCA saturation throughput, which might not be the actual throughput. The reason we use it is that the analysis for the EDCA saturation throughput is much easier and mature. The obtained voice capacity can be regarded as a lower bound.

\subsection{EDCA Saturation Throughput Analysis}

Several analytical models [15 14] have been proposed to analyze the performance of EDCA under saturation conditions, where the transmission queue of each station is assumed to be always nonempty. All of the existing EDCA modelling schemes are fundamentally based on the Bianchi's work [16], which introduces using the Markov chain to model DCF.

In our previous work [14], we have developed a simplified Markov chain model for the EDCA performance analysis, which takes not only most of the EDCA parameters but also transmission errors into consideration. Directly applying the model in this research, we calculate $S_{i}, i \in\{u p, d w\}$ according to the ratio of the time occupied by the transmitted information in a time interval to the average length of a time interval, i.e.

$$
\begin{aligned}
S_{i} & =R^{11} \frac{E[\text { time used for successful transmission in an interval }]}{E[\text { length between two consecutive transmissions }]} \\
& =R^{11} \frac{P_{i, s} E[P]}{E[I]+E[N C]+E[C]}
\end{aligned}
$$

where $R^{11}$ is the physical transmission rate of 802.11, $E[P]$ is the VoIP payload length, $P_{i, s} E[P]$ is the average amount of successfully transmitted payload information, and the average length of a time interval consists of three parts: $E[I]$, the expected value of idle time before a transmission, $E[N C]$, transmission time without collision, and $E[C]$, collision time. The details of the derivation can be found in [14].

\subsection{16 Throughput Analysis}

Considering only one SS attached to a BS in 802.16, we calculate the time length of one frame as

$$
\begin{array}{r}
T_{\text {Frame }}^{16}=T_{\text {LongPre }}^{16}+T_{F C H}^{16}+T_{D \text { Lburst }}^{16}+T_{T T G}^{16}+ \\
T_{\text {InitRang }}^{16}+T_{B \text { Wrequest }}^{16}+T_{\text {ULburst }}^{16}+T_{R R G}^{16}
\end{array}
$$

where each term corresponds to one component in the frame structure shown in Fig. 3 The terms $T_{D \text { Lburst }}^{16}$ and $T_{U L b u r s t}^{16}$ are further divided into

$$
\begin{gathered}
T_{\text {DLburst }}^{16}=T_{\text {ULburst }}^{16}=T_{\text {Pre }}^{16}+T_{M A C}^{16}+T_{\text {Pad }}^{16} \\
T_{M A C}^{16}=T_{\text {header }}^{16}+T_{\text {subheader }}^{16}+L / R^{16}+T_{C R C}^{16},
\end{gathered}
$$

where $L$ is the payload length in bits. The particular parameter values defined in 802.16 are [17]: six bytes for header, four bytes for CRC, three ranging slots with each slot corresponding to eight OFDM symbols, ten bandwidth request slots with each slot corresponding to two OFDM symbols, two OFDM symbols for TTG and RTG, two OFDM symbols for the Preamble at the frame head of frame, and one OFDM symbol for the PDU Preamble. 


\section{Numerical Results}

For experiments, we adopt the system parameters of the 802.11a and 802.16a physical layers. For EDCA, we set $W_{u p}=32, A I F S[u p]=A I F S[d w]=2, C W \max [u p]=$ $C W \max [d w]=1023$ and a maximum retry limit of 7 . We consider $\mathrm{G} .711$ voice codec is used in the application layer with a packetization interval of $20 \mathrm{~ms}$, a raw voice packet is 160 bytes. From the viewpoint of the MAC layer, the frame payload size is $160+40=200$ bytes and the data rate is $200 \times 8 / 20=80 \mathrm{kbps}$.

First, we assume the physical data rates for 802.16 and 802.11 are $6.91 \mathrm{Mbps}$ and 6 Mbps, respectively. We compare our proposed scheme that use priority and cooperation with two other schemes, where one has no priority and the other has no cooperation. The throughput performance for our proposed scheme is shown in Fig. 5, which depict the aggregate one-way voice traffic load, the aggregate 802.11 uplink throughput and the 802.11 downlink throughput. Note that the 802.16 uplink and downlink throughput is equal to the aggregate one-way voice traffic load according to our system setup. We would also like to point out that, in Fig. 6, when the number of voice connections is small, the throughput is larger than the input traffic load, which is not realistic. This is because the throughput we plot is the saturation throughput while the cases of small numbers of voice connections are actually under unsaturation conditions. From the figure, we can see that, when $W_{d w}=2$, the number of supported voice connections is 12 , beyond which either the 802.11 uplink throughput or the downlink throughput will become less than the traffic load. If we increase $W_{d w}$ to three, we will increase the number of supported voice connections to 14 . However, if we further increase $W_{d w}$ to 4 or larger, the 802.11 downlink throughput will decrease, which leads to a reduced number of supported voice connections. Therefore, $W_{d w}=3$ should be the optimal solution and $N=14$ is the maximum number of supported voice connections.

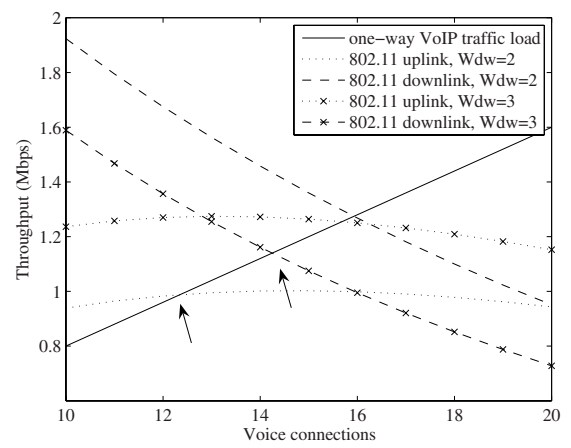

Fig. 5. The throughput performance for our proposed scheme using priority and cooperation

For the scheme without priority, we set $W_{d w}=W_{u p}=32$. Fig. 6 shows its throughput performance. It can be seen that the maximum number of supported voice connections in this situation is about five, which is much less than our proposed scheme. This is because without priority the AP becomes the bottleneck for the communications in 
802.11. For the scheme without cooperation, we fix the resource allocation between 802.16 and 802.11 to $50 \%$, i.e. $r=0.5$. Fig. 7 shows the throughput performance. It can be seen that the maximum number of supported voice connections in this situation is about 11 , which is slightly smaller than the optimal result. However, such a fixed resource allocation could lead to much worse performance since it does not consider the actual traffic conditions in 802.11 and 802.16 networks. On the contrary, our cooperation mechanism dynamically adjust $r$ according to the traffic loads, which effectively allocates the resource between 802.11 and 802.16.

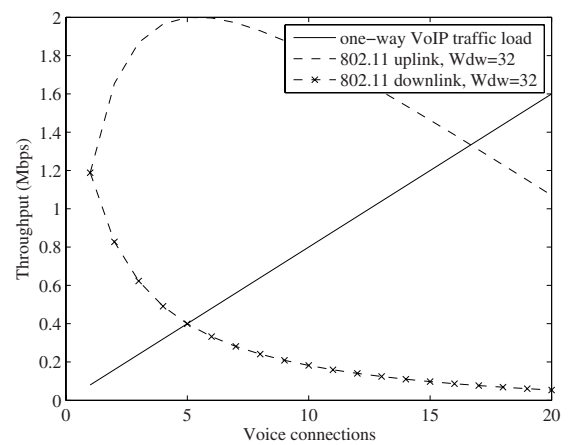

Fig. 6. The throughput performance for the scheme without using priority

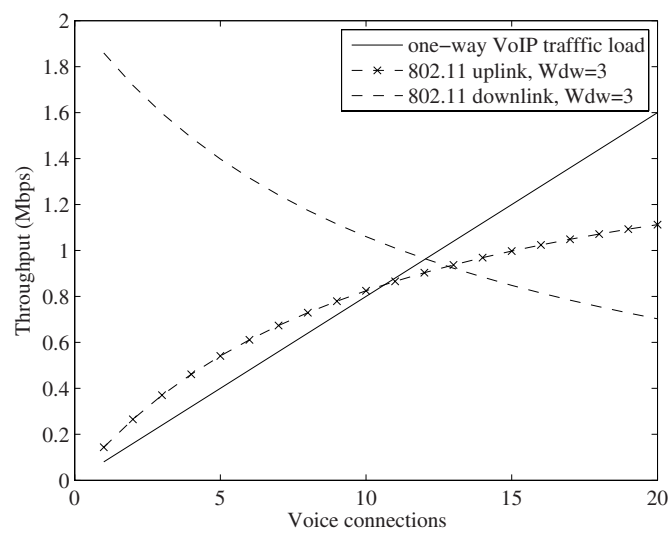

Fig. 7. The throughput performance for the scheme without cooperation

To consider different channel conditions, we vary the 802.16 data rate while fixing the 802.11 data rate to $6 \mathrm{Mbps}$. Table 1 shows the maximum numbers of supported voice connections under different 802.16 PHY-layer modes. We can see that when the 802.16 data rate is low, it has great impact on the system performance. On the other hand, when the data rate becomes much higher than the 802.11 data rate, it has affect the voice 
capacity at all. This is because when the 802.16 data rate is high, the resource percentage it needs becomes very small and the voice capacity soly depends on the performance of 802.11. Similar observations in Table 2 can be made when we fix the 802.16 data rate and vary the 802.11 data rate. However, the reason behind this phenomena is different. In 802.11a WLANs, the physical and MAC overheads are fixed for each frame and the transmission rate variation has no impact on these overheads. The VoIP frame payload which is small has few impact on the total transmission time of each frame when the transmission rate is large. Therefore, the number of stations that the system can support varies rarely when the 802.11 a transmission rate becomes higher.

Table 1. The maximum numbers of supported voice connections under different 802.16 PHYlayer modes

\begin{tabular}{|c|c|c|c|c|c|}
\hline Modulation & Code Rate & $\begin{array}{c}\text { Data Rate } \\
\text { (Mbps) }\end{array}$ & $\begin{array}{c}\text { Max. } \\
\text { Voice Conn. }\end{array}$ & $W_{d w}$ & $r$ \\
\hline BPSK & $1 / 2$ & 6.91 & 14 & 3 & 0.343 \\
\hline QPSK & $1 / 2$ & 13.82 & 16 & 3 & 0.205 \\
\hline QPSK & $3 / 4$ & 20.74 & 18 & 2 & 0.158 \\
\hline 16 QAM & $1 / 2$ & 27.65 & 20 & 2 & 0.135 \\
\hline 16 QAM & $3 / 4$ & 41.47 & 21 & 2 & 0.096 \\
\hline 64 QAM & $2 / 3$ & 55.3 & 21 & 2 & 0.077 \\
\hline 64 QAM & $3 / 4$ & 62.21 & 21 & 2 & 0.070 \\
\hline
\end{tabular}

Table 2. The maximum numbers of supported voice connections under different 802.11a PHYlayer modes

\begin{tabular}{|c|c|c|c|c|c|}
\hline Modulation & Code Rate & $\begin{array}{c}\text { Data Rate } \\
(\mathrm{Mbps})\end{array}$ & $\begin{array}{c}\text { Max. } \\
\text { Voice Conn. }\end{array}$ & $W_{d w}$ & $r$ \\
\hline BPSK & $1 / 2$ & 6 & 14 & 3 & 0.343 \\
\hline BPSK & $3 / 4$ & 9 & 16 & 2 & 0.390 \\
\hline QPSK & $1 / 2$ & 12 & 19 & 2 & 0.459 \\
\hline QPSK & $3 / 4$ & 18 & 22 & 2 & 0.528 \\
\hline 16 QAM & $1 / 2$ & 24 & 23 & 2 & 0.552 \\
\hline 16 QAM & $3 / 4$ & 36 & 24 & 2 & 0.575 \\
\hline 64 QAM & $2 / 3$ & 48 & 25 & 2 & 0.598 \\
\hline 64 QAM & $3 / 4$ & 54 & 25 & 2 & 0.598 \\
\hline
\end{tabular}

\section{Conclusion}

In this paper, we proposed the use of CAPs for 802.16 communications and CPs for 802.11 communications in the hybird 802.16/802.11 networks. We have analyzed the resource allocation issues for VoIP over the hybrid networks. By carefully choosing the $W_{d w}$, we are able to give the AP more priorities than the stations in 802.11, which solves the bottleneck problem in VoIP applications. By adjusting $r$, we are able to dynamically adjust the resource allocation between 802.16 and 802.11. Preliminary results have shown the significant improvement in voice capacity. 


\section{Acknowledgments}

The authors gratefully acknowledge the support of the Research Fund of Beijing Jiaotong University under Grant No. 2007RC017, and the support of the National High Technology Research and Development Program of China (863 program) under Grant No. 2007AA01Z241.

\section{References}

1. Mitola, I.J., Maguire, J.G.Q.: Cognitive radio: making software radios more personal. IEEE Wireless Commun. Mag. 6(4), 13-18 (1999)

2. Jesuale, N.: Spectrum policy issues for state and local government. International Journal of Network Management 16(2), 89-101 (2006)

3. Haykin, S.: Cognitive radio: brain-empowered wireless communications. IEEE J. Select. Areas Commun. 23(2), 201-220 (2005)

4. Xing, Y., Chandramouli, R., Mangold, S., S.N.: Dynamic spectrum access in open spectrum wireless networks. IEEE J. Select. Areas Commun. 24(3), 626-637 (2006)

5. Garuda, C., Ismail, M.: A multiband CMOS RF front-end for 4G WiMAX and WLAN applications. In: Proc. 2006 IEEE International Symposium on Circuits and Systems (ISCAS 2006), Island of Kos, Greece, May 2006, pp. 3049-3052 (2006)

6. Intel Corporation, Understanding Wi-Fi and WiMAX as metro-access solutions, Intel White Paper, [Online] (2004), http://www. intel.com/netcomms/technologies/wimax/304471.pdf

7. Berlemann, L., Hoymann, C., Hiertz, G., Walke, B.: Coexistence of IEEE 802.16 and 802.11(a) in unlicensed frequency bands. In: Proc. the 16th Meeting of Wireless World Research Forum (WWRF16), Shanghai, China (April 2006)

8. Jing, X., Mau, S.-C., Raychaudhuri, D., Matyas, R.: Reactive cognitive radio algorithms for co-existence between IEEE 802.11b and 802.16a networks. In: Proc. IEEE Global Telecommunications Conference (GLOBECOM 2005), St Louis, MO, November 2005, vol. 5, pp. 2465-2469 (2005)

9. Jing, X., Raychaudhuri, D.: Spectrum co-existence of IEEE 802.11b and 802.16a networks using the CSCC etiquette protocol. In: Proc. First IEEE International Symposium on New Frontiers in Dynamic Spectrum Access Networks (DySPAN 2005), Baltimore, Maryland USA, November 2005, pp. 243-250 (2005)

10. Wireless LAN Medium Access Control (MAC) and Physical Layer (PHY) Specifications, IEEE Std. 802.11 (1999)

11. Wireless LAN Medium Access Control (MAC) and Physical Layer (PHY) specifications Amendment 8: Medium Access Control (MAC) Quality of Service Enhancements, IEEE Std. 802.11e-2005 (2005)

12. IEEE Standard for Local and metropolitan area networks Part 16: Air Interface for Fixed and Mobile Broadband Wireless Access Systems, IEEE Std. 802.16e-2004 (2004)

13. Cicconetti, C., Lenzini, L., Mingozzi, E., Eklund, C.: Quality of service support in IEEE 802.16 networks. IEEE Network 20(2), 50-55 (2006)

14. Gao, D., Cai, J., Chen, C.W.: Capacity analysis of supporting VoIP in IEEE 802.11e EDCA WLANs. In: Proc. the 49th IEEE Global Telecommunications Conference (GLOBECOM 2006), San Francisco, California (November 2006) 
15. Kong, Z., Tsang, D., Bensaou, B., Gao, D.: Performance analysis of IEEE 802.11e contention-based channel access. IEEE J. Select. Areas Commun. 22(10), 2095-2106 (2004)

16. Bianchi, G.: Performance analysis of the IEEE 802.11 distributed coordination function. IEEE J. Select. Areas Commun. 18(3), 535-547 (2000)

17. Hoymann, C.: Analysis and performance evaluation of the OFDM-based metropolitan area network IEEE 802.16. Computer Networks 49(3), 341-363 (2005) 